\title{
Evidential Support and Instrumental Rationality
}

\author{
Peter Brössel, Anna-Maria A. Eder, and Franz Huber \\ Formal Epistemology Research Group \\ Zukunftskolleg and Department of Philosophy \\ University of Konstanz \\ Germany \\ penultimate version: please cite the paper in \\ Philosophy and Phenomenological Research - Franz Huber \\ $07 / 07 / 2014$
}

\section{Introduction}

An attractive view of epistemic rationality classifies it as a species of instrumental rationality according to which rationality consists in taking the means to an end. For instance, Quine (1986) seems to support this view when he writes that

[...] normative epistemology is a branch of engineering. It is the technology of truth-seeking [...] [I]t is a matter of efficacy for an ulterior end, truth [...] The normative here, as elsewhere in engineering, becomes descriptive when the terminal parameter is expressed. (Quine 1986: 664-665)

In a recent paper Thomas Kelly (2003) criticizes this view. For him epistemic rationality is 
roughly, the kind of rationality which one displays when one believes propositions that are strongly supported by one's evidence and refrains from believing propositions that are improbable given one's evidence. (Kelly 2003: 612)

Kelly holds that epistemic rationality cannot be understood as a form of instrumental rationality, because an agent may lack the relevant cognitive goal while still being epistemically rational. Suppose an agent does not have the cognitive goal of believing the truth or has other, overriding goals. In this case it is not necessarily instrumentally rational for her to believe a certain proposition even if the latter is strongly supported by her evidence, and thus, on Kelly's view, a proposition which it would be epistemically rational for her to believe.

In his reply Adam Leite (2007) points out a way the instrumentalist conception of epistemic rationality can be defended. He distinguishes

questions about what the evidence supports from questions about what a person ought to believe. (Leite 2007: 456)

By tying epistemic rationality to what a person ought or, as Kelly (2007: 467) puts it, has reason to believe rather than to what her evidence supports the instrumentalist conception of epistemic rationality can be saved.

In his reply Kelly argues that this way out is not viable, because the relevant notion of evidence or evidential support is normative. Therefore, Kelly claims, "there is no gap between possessing evidence that some proposition is true and possessing reasons to think that that proposition is true" (Kelly 2007: 468). In other words, Kelly seems to think that if evidential support is a normative notion, then an agent ought, or has reason, to believe a proposition if and only if that agent's evidence supports that proposition.

In this paper we claim, with Leite and against Kelly, that an agent's possessing evidence that supports a proposition and that agent's de facto having reason to believe that that proposition is true come apart when the agent does not have the relevant epistemic goal of believing the truth about that proposition. This is so despite the fact that evidential support is a normative notion, for it is a hypothetically, as opposed to categorically, normative 
notion. Accordingly we hold that epistemic rationality is instrumental rationality given the goal of believing the truth: an agent's belief in a proposition is epistemically rational if and only if that agent has reason to believe that proposition given she has the goal of believing the truth about that proposition.

We also claim, with Kelly and against Leite, that epistemic rationality is tied to what the evidence supports rather than to what one has reason to believe. Believing a proposition that is supported by one's evidence is instrumentally rational for believing the truth about that proposition.

Finally we show that the instrumentalist conception of epistemic rationality can be saved, because epistemic rationality is tied to evidential support, evidential support is hypothetically normative, and therefore epistemic rationality is a form of instrumental rationality.

For ease of presentation we start by considering only individual beliefs and the question of their instrumental rationality. Specifically, we do not consider the case of two or more beliefs, let alone the case of belief systems. In the final section we drop this restriction and consider global goals concerning an agent's belief system in order to correctly state our positive arguments.

First, however, let us hear what Kelly and Leite have to say about evidential support and instrumental rationality.

\section{$2 \quad$ Kelly and Leite on Evidential Support and Instrumental Rationality}

For Kelly being epistemically rational is tantamount to believing what one's evidence supports, which in turn is tantamount to believing what one has reason to believe. Call this Kelly's trinity (Figure 1).

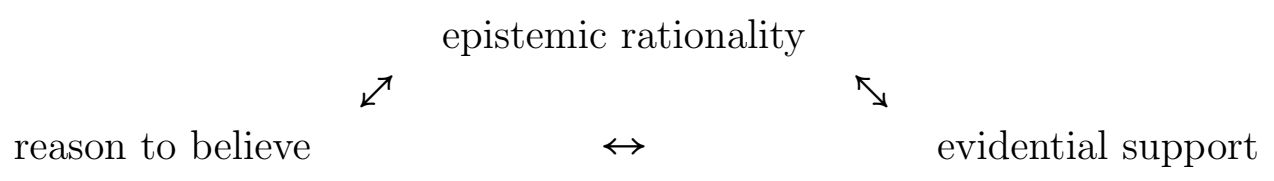

Figure 1 
Kelly's trinity consists of three claims. The first claim (I) equates epistemically rational beliefs with beliefs supported by one's evidence. The second claim (II) equates beliefs supported by one's evidence with beliefs one has reason to believe. The third claim (III) equates epistemically rational beliefs with beliefs one has reason to believe. ${ }^{1}$

(I) epistemic rationality $\leftrightarrow$ evidential support

(II) evidential support $\leftrightarrow$ reason to believe

(III) epistemic rationality $\leftrightarrow$ reason to believe

(III) is an implicit claim in Kelly (2003) insofar as he does not seem to distinguish between beliefs that are epistemically rational and beliefs that one has reason to believe. (I) is an explicit claim insofar as epistemic rationality is characterized in terms of what the evidence supports (Kelly 2003: 612). (II) is a consequence of (I) and (III).

This picture of the relation between epistemic rationality, evidential support, and reason to believe leads Kelly to doubt the instrumentalist conception of epistemic rationality. According to Kelly (2003: 621) "the most serious reason for skepticism about the instrumentalist conception of epistemic rationality is this: what a person has reason to believe does not seem to depend on the content of his or her goals in the way that one would expect if the instrumentalist conception were correct". Kelly assumes that what evidence a person possesses and which propositions this evidence supports does not depend on the goals this person actually has. Since Kelly (2003: 612) presupposes that epistemic rationality is "the kind of rationality which one displays when one believes propositions that are strongly supported by one's evidence" he concludes that epistemic rationality does not depend on the content of a person's goals.

In support of his view Kelly (2003: 621) notes that we ordinarily talk as if reason to believe was categorical reason to believe. Another reason, Kelly thinks, is "that one can have epistemic reasons to believe propositions even in cases in which it is clear that one's believing those propositions holds no promise of advancing any goal which one actually has" (Kelly 2003: 630).

\footnotetext{
${ }^{1}$ Kelly $(2003 ; 2007)$ and Leite (2007) mostly use the terms 'epistemic reason', 'reason to believe', and 'epistemic reason to believe' synonymously.
} 
For Leite Kelly is begging the question when he characterizes epistemically rational beliefs as beliefs supported by one's evidence.

The instrumentalist need not accept that this is a kind of rationality at all-except insofar as one has the goals (whatever they might be) which would make such behavior instrumentally rational. (Leite 2007: 457)

On behalf of the instrumentalist Leite suggests separating the question of what one has reason to believe from the question of what is supported by one's evidence (Leite 2007: 456). In terms of Kelly's trinity he denies (I) as well as (II).

Having epistemic reasons to believe that $p$ is having epistemic reasons to do something: to form a belief on a certain subject matter. But the mere possession of strong evidence doesn't give me a reason to form a belief. There are many propositions which it would be pointless for me to bother to take any attitude towards, even though I possess strong evidence in their favor. I can't justly be charged with irrationality - even with epistemic irrationality - for failing to do so. (Leite 2008: 561)

Leite subscribes to (III) and contends that one can defend the instrumentalist conception of epistemic rationality by characterizing the latter in terms of reason to believe rather than evidential support. Call the result Leite's duality (Figure 2).

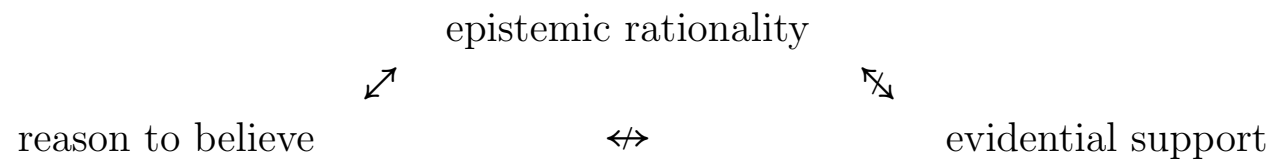

Figure 2

Leite's rejection of (II) is a natural picture to have on a non-normative view of evidential support. For instance, if evidential support is defined in terms of a frequentist notion of reliability, then what is supported by one's evidence may come apart from what one has reason to believe, if only 
because one is not aware of the obtaining evidential relationships. According to both Kelly and Leite the notion of evidential support required by the instrumentalist and standing in the requisite relation to reason to believe "functions as a kind of non-normative substrate for reasons for belief: in cases in which one does have reasons to think that some proposition is true, this is so in part because one has evidence that the proposition is true and in part because one possesses some appropriate goal" (Kelly 2007: 469).

Kelly (2007: 470) agrees with Leite (2007) that there are non-normative notions of evidential support, but points out that they do not function in the way required by the instrumentalist. For instance, if evidential support is defined in terms of a frequentist notion of reliability, then we have a nonnormative notion of evidential support, but that notion does not function in the required way: the external evidential relationship may obtain and one may possess the appropriate goal of believing the truth, and yet one does not have reason to believe that some proposition is true if one is not aware that the external evidential relationship obtains. Kelly goes on to note that he does not know of an adequate non-normative notion of evidential support that functions in the required internal way, and doubts that there is one. In support of the conjecture that there is none Kelly mainly argues on the basis of the intuitive (im)plausibility of various examples. One of these proceeds as follows. If evidential support and reason to believe can come apart, as Leite holds, then "statements such as the following should have no whiff of paradox":

I have overwhelming evidence that $p$ is true. But I have no reason to think that $p$ is true.

For Kelly the paradoxical character of such statements is best explained by evidential support being a normative notion. For him "there is no gap between possessing evidence that some proposition is true and possessing reasons to think that that proposition is true" (Kelly 2007: 468), if evidential support is a normative notion. Recall that both Kelly and Leite use 'has reason to believe' and 'ought to believe' interchangeably: an agent has reason to believe a proposition if and only if that agent ought to believe that proposition. Kelly seems to think that, if evidential support is a normative 
notion, then an agent ought to believe a proposition if and only if that agent's evidence supports that proposition.

Kelly (2007: 471) admits that his argumentation does "not show that there is no non-normative notion [of evidential support] that stands in the requisite relation to the normative concept of a reason for belief and is thus suitable for the instrumentalist's purposes". However, he holds, "it would be unwarranted to simply assume that there must be some such thing". He concludes by locating the burden of proof on the side of the instrumentalist.

Below we discharge the burden of proof and show that a notion of evidential support in epistemology and the philosophy of science is some such thing. More precisely, we will show that this notion fits the instrumentalist's bill by being hypothetically normative. First, however, let us present our view of the relation between rationality, reason to believe, and evidential support.

\section{Evidential Support and Instrumental Ra- tionality}

In this section we merely present and illustrate our view of the relation between rationality, reason to believe, and evidential support without positively arguing for its adequacy.

We propose to replace Kelly's trinity with our trinity a (Figure 3).

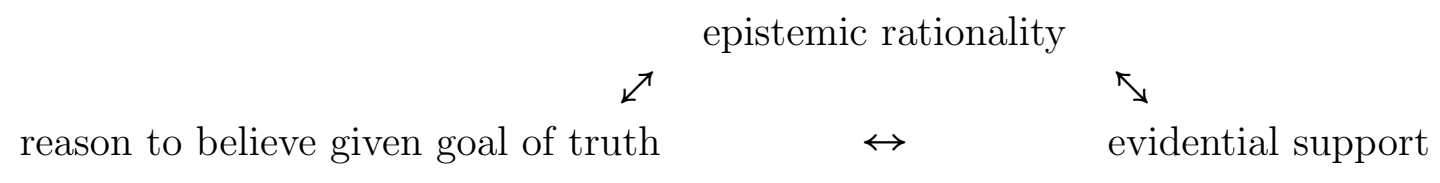

Figure 3

We agree with Kelly's claim (I), which equates epistemically rational beliefs with beliefs supported by one's evidence. More specifically, we subscribe to claim (Ia) that it is epistemically rational for an agent to believe a proposition if and only if that agent's evidence supports that proposition.

(Ia) epistemic rationality $\leftrightarrow$ evidential support 
We agree with Leite's rejection of claim (II), which equates beliefs supported by one's evidence with beliefs one has reason to believe. On our view supporting evidence does not provide a categorical reason to believe. Supporting evidence only provides a hypothetical reason to believe conditional on the goal of believing the truth, or a reason to believe given the goal of believing the truth. Kelly assumes that an "instrumental reason is a hypothetical reason, in the sense that it depends for its existence on the fact that the individual for whom it is a reason possesses a certain goal or goals" (Kelly 2003: 621). Contrary to Kelly we do not assume that hypothetical reasons may only depend on the agent's actual goals or, as we prefer to say, her de facto goals. On our view the "existence" of an instrumental reason does not depend on whether an individual has the appropriate goal. The instrumental reason "exists" just in case the appropriate means-end relationship obtains. Whether an "existing" instrumental reason is also an actual reason for an agent or, as we prefer to say, a de facto reason for an agent depends on that agent's de facto goals.

Restricted to the present epistemological context we call a norm that holds, and holds only, conditional on a goal a hypothetical norm. A hypothetical norm conditional on some goal is normative insofar as it tells one what one ought to do given that one has the goal at issue. A hypothetical norm conditional on some goal does not tell one what one ought to do if one does not have the goal at issue, let alone what one ought to do unconditionally. Such latter norm we call categorical. A hypothetical norm conditional on a goal holds just in case there exists a means-end relationship between the norm in question and the goal at issue. A hypothetical norm conditional on a goal is descriptive insofar as means-end relationships between the norm in question and the goal at issue can be described by propositions that are true or false. One justifies a hypothetical norm conditional on a goal by establishing that the norm in question is a means to attaining the goal at issue.

Let us illustrate our view with an analogy. We take it that financial reasons are paradigmatic examples of instrumental reasons. The existence of a financial reason to buy a lottery ticket does not depend on an agent's de facto goals. A financial reason to buy a lottery ticket exists just in case 
the appropriate means-end relationship obtains. On the standard view the financial reason to buy a lottery ticket exists just in case buying the lottery ticket maximizes the agent's expected financial gain. Whether an existing financial reason to buy a lottery ticket is a de facto reason for an agent depends on that agent's de facto goals. Importantly, the existence of a financial reason to buy a lottery ticket does not imply that the agent has the goal of maximizing her expected financial gain. Epistemic reasons to believe behave analogously. The existence of an epistemic reason to believe a proposition does not depend on an agent's de facto goals. An epistemic reason to believe a proposition exists just in case the appropriate means-end relationship obtains. On a view that seems to be compatible with Kelly's (see the quote and the presentation of Kelly's view on the first page of this paper), the epistemic reason to believe a proposition exists just in case believing the proposition is (subjectively) probable to result in believing the truth about the proposition. Whether an existing epistemic reason to believe a proposition is a de facto reason for an agent depends on that agent's de facto goals. Importantly, the existence of an epistemic reason to believe a proposition does not imply that an agent has the goal of believing the truth about that proposition.

It is interesting to ask why and how the existence of an instrumental reason to take some action is relevant if it does not guarantee that there exists a de facto reason for the agent to take that action.It is equally interesting to ask why and how a hypothetical norm is relevant if it does not tell an agent what she ought to do.

On our view an agent has a de facto reason to take some action if and only if the appropriate means-end relationship between the action and the end obtains, and the end belongs to the agent's de facto goals. Therefore the existence of an instrumental reason is relevant, because the agent's de facto possessing the appropriate goal turns a merely hypothetical reason into a de facto reason for the agent. Similarly, a hypothetical norm is relevant, because the agent's de facto possessing the appropriate goal turns a merely hypothetical norm into a de facto norm for the agent, thus telling her what she de facto ought to do.

Instead of (II) we hold that beliefs supported by one's evidence coincide with beliefs one has reason to believe given the goal of believing the 
truth. More specifically, we subscribe to claim (IIa) that an agent's evidence supports a proposition if and only if that agent has reason to believe that proposition given she has the goal of believing the truth about it.

(IIa) evidential support $\leftrightarrow$ reason to believe given goal of truth

Furthermore, we disagree with both Kelly and Leite and reject their claim (III), which equates epistemically rational beliefs with beliefs one has reason to believe. Again, on our view the evidence does not provide a categorical reason to believe. Instead of (III) we hold that epistemically rational beliefs coincide with beliefs one has reason to believe given the goal of believing the truth. More specifically, we subscribe to claim (IIIa) that it is epistemically rational for an agent to believe a proposition if and only if that agent has reason to believe that proposition given she has the goal of believing the truth about it.

(IIIa) epistemic rationality $\leftrightarrow$ reason to believe given goal of truth

Both Kelly and Leite correctly point out that we sometimes do not aim at believing the truth about some proposition. Kelly's (2003: 626) example of the movie, where he "make[s] a conscious, deliberate effort to avoid finding out how the movie ends" before viewing the movie, might be a case in point. We think it is only an example of an agent trying to come to believe the truth about a proposition in a particularly entertaining way. We do not even want to exclude that an agent aims at believing the falsity about a proposition.

Believing the truth is not a universal goal in the sense that everybody has it at any time with respect to every proposition. It is, however, a pervasive and important goal. Indeed, it is the default cognitive goal. In that respect believing is similar to asserting. The default goal of asserting is asserting the truth, and the default goal of believing is believing the truth. However, just as we sometimes use assertions to comfort, deceive, encourage, and entertain others, independently of whether the assertions are true or false, we sometimes use beliefs to comfort, deceive, encourage, and entertain ourselves, independently of whether the beliefs are true of false. A grieving mother may 
aim at believing that her deceased son was a good person in order to comfort herself, independently of whether her son in fact was a good person and, hence, not necessarily in order to believe the truth about whether her son was a good person.

Believing the truth is the default cognitive goal. This explains the paradoxical character of statements such as:

I have overwhelming evidence that $p$ is true. But I have no reason to think that $p$ is true.

It also explains why we use 'rational belief' as shorthand for 'epistemically rational belief', and why we ordinarily treat reasons to believe as categorical reasons, both "in offering such reasons to others" and "in responding to such reasons in the course of one's own deliberation" (Kelly 2003: 621). In interpersonal discourse we assume the participants to adopt the default goal of believing the truth. Unless specified otherwise, we assume that it is relevant evidence that is offered to us as reason to believe, and we assume that we are expected to provide relevant evidence as reason to believe. The assumption of the default goal of believing the truth is not appropriate in all contexts, though.

If one's de facto goal with respect to some proposition is not believing the truth about that proposition, then the reason to believe that proposition given the goal of believing the truth about it may still exist. The reason is that the appropriate means-end relationship obtains or does not obtain independently of one's de facto goals. If the appropriate means-end relationship obtains and one's de facto goal with respect to the proposition in question is not believing the truth about that proposition, then epistemic rationality and actual rationality or, as we prefer to say, de facto rationality come apart. The claim (IIIb) that we subscribe to is that it is de facto rational for an agent to believe a proposition if and only if that agent has reason to believe that proposition given her de facto goals. ${ }^{2}$ This in turn holds just in case

\footnotetext{
${ }^{2}$ More generally, we subscribe to the claim (III ${ }^{*}$ ) that, for all combinations of goals, $X$, it is $X$-rational for an agent to believe a proposition if and only if that agent has reason to believe that proposition given she has goals $X$.
} 
the appropriate means-end relationship between the proposition in question and the agent's de facto goals with respect to that proposition obtains.

(IIIb) de facto rationality $\leftrightarrow$ reason to believe given de facto goals

Consequently we propose to replace Leite's duality with our duality $\boldsymbol{b}$ (Figure 4).

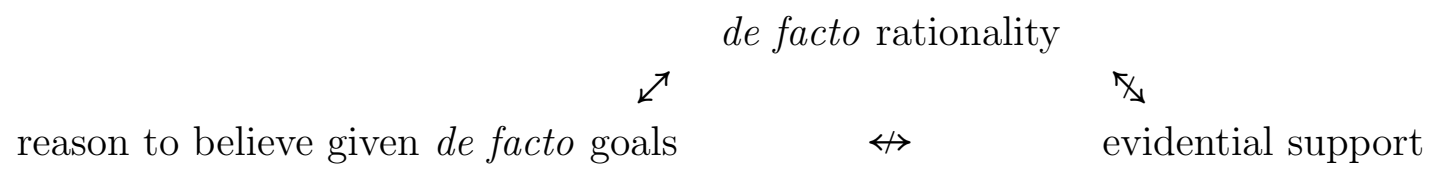

Figure 4

Thus, if one's de facto goal with respect to some proposition is not believing the truth about that proposition, then there may be a gap between possessing evidence that supports that proposition and having a de facto reason to believe that it is true. ${ }^{3}$

For purposes of illustration consider the following stock example. A man has evidence that strongly supports that his wife is unfaithful. However, he loves his wife so much that, as he knows, his de facto goals are best served if he believes that she is faithful, no matter whether or not she in fact is. If this man believed that his wife is unfaithful, his belief would be supported by his evidence, even though he would not have reason to believe so given his de facto goals. If this man achieved believing that his wife is faithful, his belief would not be epistemically rational, but de facto rational. Love may be blind, but love need not be irrational!

(III $\left.{ }^{*}\right) \quad X$-rationality $\leftrightarrow \quad$ reason to believe given goals $X$

What we have called epistemic rationality in our trinity a may thus be called truthrationality. However, we continue to follow Kelly and Leite in their use of 'epistemic rationality'. On our view claim $\left(\mathbf{I I I}^{*}\right)$ is true, because ' $X$-rationality' is synonymous with 'reason to believe given goals $X$ '.

${ }^{3}$ We do not exclude that there are collective goals of groups of agents, as opposed to goals of individual agents, nor that there are objectively valid goals, as opposed to subjectively desired goals. 
Of course, in examples such as these it often happens that the agent involuntarily comes to believe the target proposition which is strongly supported by her evidence. However, the question of voluntarism should be separated from the question of the instrumentalist conception of epistemic rationality. Maybe the following example brings out the difference between epistemic rationality and de facto rationality more clearly. Suppose the man who loves his wife so much and who has evidence that strongly supports that his wife is unfaithful involuntarily comes to believe that his wife is unfaithful and, as a consequence, becomes depressive. Suppose further his de facto goals include curing his depressions and, as he knows, are best served if he represses his belief that his wife is unfaithful. If, by therapeutic means, say, this man achieved repressing his belief that his wife is unfaithful (and only this belief without also the evidence strongly supporting it), he would not be epistemically rational, but de facto rational.

Kelly (2003: 632-634) discusses a strategy such as ours. According to him distinguishing the possibly hypothetical goal of believing the truth about a proposition and an agent's de facto goals "is not enough to save the instrumentalist conception of epistemic rationality. The crucial fact here is the following: whether it is in fact instrumentally rational for me to $\Phi$ depends on the content of the goals which I actually hold. [...] Only goals which I actually hold make a difference to what is instrumentally rational for me."

By definition, instrumental rationality is rationality with respect to some goals. If, on the one hand, the goals with respect to which one judges an agent to be instrumentally rational are that agent's de facto goals, then Kelly's claim is true, but besides the point. Recall that epistemic rationality and de facto rationality may come apart if the agent's de facto goal with respect to some proposition is not believing the truth about that proposition. So far we agree. However, on our view the existence of an instrumental reason does not depend on whether an individual has the relevant goal. The instrumental reason exists just in case the appropriate means-end relationship obtains. Whether an instrumental reason is a de facto reason for an agent depends on that agent's de facto goals.

If, on the other hand, the goals with respect to which one judges an agent to be instrumentally rational are not that agent's de facto goals, then 
Kelly's claim is false. The good man whose de factogoals are best served if he donates money in such a way that he will never financially profit from it is not financially rational, but de facto rational. It does not follow that financial rationality cannot be understood in instrumentalist terms.

\section{Evidential Support Revisited}

Let us now discharge the burden of proof and show that a notion of evidential support in epistemology and the philosophy of science is a hypothetically normative "notion that stands in the requisite relation to the normative concept of a reason for belief and is thus suitable for the instrumentalist's purposes" (Kelly 2007: 471).

\subsection{Evidential Support and Degrees of Belief}

In a footnote following the above quote Kelly (2007: 471) refers to Carnap's (1962) notion of degree of confirmation, which is a concept of evidential support that would be suitable for the instrumentalist's purposes. The reason is that it is "a wholly non-normative, logical relation" and so "the instrumentalist might be in a position to simply identify epistemic rationality with the instrumental rationality of employing such a logic".

Kelly correctly points out that Carnap's project can plausibly be considered to have failed and that contemporary Bayesians typically define evidential support in terms of the normative notion of a rational degree of belief function. Therefore the notion of evidential support is itself normative and so, Kelly thinks, cannot be related to the normative notion of reason to believe in the way required by the instrumentalist. (Similarly on page 467, where he claims that any standard Bayesian notion of evidential support is normative.) Thus, he claims, a Bayesian notion of evidential support is not suitable for the instrumentalist's purposes.

We disagree. Bayesians have two notions of evidential support. Absolute evidential support is defined as conditional probability in the sense of a probability measure Pr, which is interpreted as the agent's rational degree of belief function: $r$ is the agent's degree of absolute evidential support for 
a proposition $h$ provided by the agent's evidence $e$ if and only if $r$ is the agent's rational degree of belief for $h$ given $e, r=\operatorname{Pr}(h \mid e)$. Incremental evidential support is defined as positive probabilistic relevance in the sense of a probability measure $\mathrm{Pr}$, which is interpreted as the agent's rational degree of belief function: $s$ is the agent's degree of incremental evidential support for a proposition $h$ provided by the agent's evidence $e$ if and only if $s$ is the difference between the agent's rational degree of belief for $h$ given $e$ and her rational degree of belief for $h, s=\operatorname{Pr}(h \mid e)-\operatorname{Pr}(h) .{ }^{4}$ For the purposes of this paper we can identify an agent's evidence with the logically strongest proposition $e$ the agent is certain of in the sense of assigning it probability $1, \operatorname{Pr}(e)=1$.

Among others because it seems to be the notion Kelly has in mind, we restrict the discussion to absolute evidential support and show that it stands in the requisite internal relation to reason to believe, as required by the instrumentalist. One of us thinks that incremental evidential support can also be put in relation to reason to believe in a way satisfying the instrumentalist, but things are more complicated here. One of the reasons why things are more complicated here is that one additionally has to consider diachronic rules for updating the agent's degree of belief function over time besides the synchronic rules for organizing the agent's degree of belief function at a given moment in time. .

Absolute evidential support is internally accessible for the agent, because it is defined exclusively in terms of the agent's rational degree of belief function. If the agent's degree of belief function is rational in the sense that it is a probability measure, and if $e$ is the agent's evidence, i.e. the logically strongest proposition the agent is certain of in the sense of assigning it probability 1 , then the agent's rational degree of belief in any proposition $h$ is her degree of absolute evidential support for $h$ given $e$. In this sense the agent believes in accordance with her evidence. Thus, whether it is rational to believe in accordance with one's evidence depends on whether probability measures really are rational degree of belief functions. This in turn depends on the existence of an appropriate means-end relationship between being a prob-

\footnotetext{
${ }^{4}$ There are different ways to measure the difference between the agent's degree of belief in $h$ given $e$ and her degree of belief in $h$. .
} 
ability measure and having true beliefs, or some other pertinent epistemic goal. Such an appropriate means-end relationship is exactly what means-end justifications attempt to establish.

The interpretation of the probability measure as the agent's rational degree of belief function results in a normative notion of evidential support, as Kelly rightly notes, since the notion of a rational degree of belief function is a normative notion. What is wrong, though, is that the normative notion of a rational degree of belief function as well as the resulting normative notion of evidential support cannot be understood in instrumentalist terms. On the contrary, ever since Ramsey (1926) philosophers have strived for a means-end justification of the normative notion of a rational degree of belief function, thus turning it into a descriptive notion by expressing the terminal parameter.

Recall that one justifies a hypothetical norm conditional on a goal by establishing that the norm in question is a means to attaining the goal at issue. The best known of these means-end justifications is the Dutch Book argument dating back to Ramsey (1926). The conclusion of the Dutch Book argument is the norm of probabilism, which is that an agent's degree of belief function should obey the probability calculus. An agent's degree of belief function is said to be Dutch Bookable if and only if there exists a series of bets all of which are individually acceptable for that agent, but together guarantee a sure loss. A bet with gain $1 \$$ on a proposition for price $r \$$ is acceptable for an agent if and only if that agent's degree of belief for that proposition is at least $r$. The core of the argument is a theorem to the effect that an agent's degree of belief function obeys the probability calculus if and only if it is not Dutch Bookable. Thus, obeying the norm of probabilism is a (provably necessary and sufficient) means to attaining the end of having a degree of belief function that is not Dutch Bookable. In other words, the norm of probabilism has a means-end justification with respect to the goal of having a degree of belief function that is not Dutch Bookable.

The Dutch Book argument does not establish a means-end relationship between the norm of probabilism and a cognitive goal, because not accepting a series of bets that guarantees a sure loss is not a cognitive goal. This is different in case of the calibration argument due to van Fraassen (1983). 
The conclusion of the calibration argument is the norm of probabilism. An agent's degree of belief function is said to be potentially calibrated if and only if there exists a possible world in which it is calibrated. An agent's degree of belief function is calibrated in a possible world if and only if, for every degree of belief $r$, the relative frequency or proportion of propositions that are true in that world among all propositions the agent believes to degree $r$ approximates $r$, where approximation summarizes some technical details that do not matter for present purposes. The idea is that calibration measures the reliability of degrees of belief as indicators of relative frequencies. The core of the argument is a theorem to the effect that an agent's degree of belief function obeys the probability calculus if and only if it is potentially calibrated. Thus, obeying the norm of probabilism is a (provably necessary and sufficient) means to attaining the end of having a potentially calibrated degree of belief function. In other words, the norm of probabilism has a means-end justification with respect to the goal of having a potentially calibrated degree of belief function.

The calibration argument does not establish a means-end relationship between the norm of probabilism and the default cognitive goal of believing the truth. This is different in case of the gradational accuracy argument (Joyce 1998, 2009). The latter has been at center stage in a recent discussion of means-end epistemology (Percival 2002, Stalnaker 2002).

The conclusion of the gradational accuracy argument is the norm of probabilism. An agent's degree of belief function is said to be accuracy dominated if and only if there exists an alternative degree of belief function that is at least as accurate in all possible worlds and more accurate in some possible world. The accuracy of an agent's degree of belief function in a possible world is determined by the accuracy of the agent's degrees of belief in all propositions in that possible world. The accuracy of the agent's degree of belief in a proposition in a possible world is the distance between the agent's degree of belief in the proposition and the truth value of the proposition in that possible world, where 1 represents truth and 0 represents falsity. A definition of the accuracy of an agent's degree of belief in a proposition in a possible world is implicitly given by axioms that say how to measure the distance between an agent's degree of belief in a proposition and the truth 
value of the proposition in that possible world. ${ }^{5}$ For instance, a degree of belief up to 1 in a proposition which is true in a possible world is more accurate in that possible world, the higher it is, and perfectly accurate if it equals 1 . The core of the argument is a theorem to the effect that an agent's degree of belief function obeys the probability calculus if and only if it is not accuracy dominated. Thus, obeying the norm of probabilism is a (provably necessary and sufficient) means to attaining the end of having a degree of belief function that is not accuracy dominated. In other words, the norm of probabilism has a means-end justification with respect to the goal of having a degree of belief function that is not accuracy dominated. Since not being accuracy dominated is a necessary condition for accuracy, which Joyce (1998: 577ff) claims to be the probabilistic counterpart of truth, the accuracy argument establishes a (provably necessary) relationship between the norm of probabilism and the goal of accuracy.

For ease of reference let us repeat the first non-categorically normative notion of evidential support that we have identified.

(E1) absolute evidential support in the sense of a probability measure Pr, which is interpreted as the agent's rational degree of belief function: $r$ is the agent's degree of absolute evidential support for a proposition $h$ provided by the agent's evidence $e$ if and only if $r$ is the agent's rational degree of belief for $h$ given $e, r=\operatorname{Pr}(h \mid e)$, where the agent's evidence $e$ is the logically strongest proposition the agent is certain of in the sense of assigning it probability $1, \operatorname{Pr}(e)=1$.

This notion of evidential support is hypothetically normative. It is defined to be identical with a probability measure which is interpreted as a rational degree of belief function. Therefore, in order to justify the norm that one ought to believe in accordance with one's evidence, it is necessary and sufficient to provide a means-end justification for the norm of probabilism. Since obeying the norm of probabilism is a provably necessary and sufficient means to attaining the end of having a degree of belief function that is not accuracy dominated, which in turn is a necessary condition for

\footnotetext{
${ }^{5}$ For the latest axiomatic definition of the accuracy of an agent's degree of belief in a proposition in a possible world see Joyce (2009).
} 
accuracy, believing in accordance with one's evidence is a provably necessary means to attaining the end of believing accurately. Thus, given that accuracy is the probabilistic counterpart of truth, believing in accordance with one's evidence is a provably necessary means to attaining the end of believing truly in a probabilistic setting, or to attaining the end of having degrees of belief in propositions that are close to the truth-values of those propositions.

The goal of not being accuracy dominated is a global goal for degree of belief functions or entire systems of degrees of belief. Therefore the gradational accuracy argument does not establish a means-end relationship between a norm for full belief about a single proposition and the local goal of believing the truth about that proposition, as in our trinity a. However, one can reformulate our trinities $\mathbf{a}$ and $\mathbf{b}$ for degrees of belief instead of full beliefs, and one can generalize these trinities to systems of degrees of belief or degree of belief functions.

\section{For degrees of belief:}

- (Ia-d) it is epistemically rational for an agent to believe a proposition to degree $r$ if and only if that agent's evidence supports that proposition to degree $r$.

- (IIa-d) an agent's evidence supports a proposition to degree $r$ if and only if that agent has reason to believe that proposition to degree $r$ given she has the goal of believing it accurately.

- (IIIa-d) it is epistemically rational for an agent to believe a proposition to degree $r$ if and only if that agent has reason to believe that proposition to degree $r$ given she has the goal of believing it accurately.

- (IIIb-d) it is de facto rational for an agent to believe a proposition to degree $r$ if and only if that agent has reason to believe that proposition to degree $r$ given her de facto goals.

Alternatively one can generalize our trinities $\mathbf{a}$ and $\mathbf{b}$ from full belief in a single proposition to systems of full beliefs. 


\section{For systems of full beliefs:}

- (Ia-s) it is epistemically rational for an agent with system of full beliefs $R$ to believe a proposition if and only if that agent's evidence supports that proposition.

- (IIa-s) the evidence of an agent with system of full beliefs $R$ supports a proposition if and only if that agent has reason to believe that proposition given she has the goal of believing the truth about it.

- (IIIa-s) it is epistemically rational for an agent with system of full beliefs $R$ to believe a proposition if and only if that agent has reason to believe that proposition given she has the goal of believing the truth about it.

- (IIIb-s) it is actually rational for an agent with system of full beliefs $R$ to believe a proposition if and only if that agent has reason to believe that proposition given her actual goals.

Then one can focus on the norm of ranking theory for systems of full beliefs (Spohn 1988, 2009) instead of the norm of probabilism for systems of degrees of belief or degree of belief functions; and one can adopt the global goal of diachronic consistency for systems of full beliefs instead of the global goal of not being accuracy dominated for systems of degrees of belief. Finally one can enter the consistency argument for ranking functions (Huber 2007) instead of the the gradational accuracy argument for probability measures.

\subsection{Evidential Support and Belief}

A function $R: \mathcal{A}_{W} \rightarrow \mathbb{N} \cup\{\infty\}$ from an algebra of propositions $\mathcal{A}_{W} \subseteq \wp(W)$ over a set of possibilities $W$ into the natural numbers $\mathbb{N}$ extended by infinity $\infty$ is a ranking function if and only if (R1) the tautological proposition is assigned rank $0, R(W)=0$, and (R2) the rank of a disjunction equals the minimum of the ranks of the disjuncts, $R(A \cup B)=\min \{R(A), R(B)\}$. Conditional ranks are defined as differences of unconditional ranks: $R(A \mid B)=$ $R(A \cap B)-R(B)$. Ranks are interpreted epistemically as grades of disbelief. A proposition $A$ is disbelieved if and only if its rank is positive, $R(A)>0$. A 
proposition $A$ is believed if and only if its negation is disbelieved, $R(\neg A)>0$. (R1) requires that the tautological proposition not be disbelieved. Part of what (R2) requires is that a disjunction be disbelieved just in case both disjuncts are disbelieved. Given the definition of conditional ranks, all (R2) requires is that a disjunction be conditionally disbelieved just in case both disjuncts are conditionally disbelieved.

(R1) and (R2) are synchronic rules for organizing one's beliefs at a given moment in time. Besides these ranking theory also includes diachronic rules for updating one's beliefs if new information of various formats is received. The simplest of these rules, corresponding to strict conditionalization in the probabilistic case and defined for the case where the new information comes in form of a certainty, is plain conditionalization. According to it an agent's new ranking function should be her old ranking function conditional on the information received. Other rules $^{6}$ are defined for the case where the new information comes in form of a partition of the space of possibilities and a set of numbers characterizing the new strengths of belief in the elements of the partition, or the degrees by which the strengths of belief in the elements of the partition have changed.

Ranking theory is centered around the categorical notion of belief, and thus much better suited for traditional philosophical purposes than probabilism, which is centered around the quantitative notion of degree of belief. Absolute evidential support is defined as conditional rank in the sense of a ranking function $R$, which is interpreted as the agent's rational system of full belief: a proposition $h$ is absolutely supported by the agent's evidence $e$ if and only if $h$ is rationally believed given $e, R(\neg h \mid e)>0$. More generally, $r$ is the agent's degree of absolute evidential support for a proposition $h$ provided by the agent's evidence $e$ if and only if $r$ is the agent's rational strength of disbelief for $\neg h$ given $e, r=R(\neg h \mid e){ }^{7}$ For the purposes of this paper we can identify an agent's evidence with the logically strongest proposition $e$ the agent is certain of in the sense of assigning its negation rank $\infty$,

\footnotetext{
${ }^{6}$ These other rules correspond to Jeffrey and Field conditionalization in the probabilistic case. See Jeffrey (1983) and Field (1978) and Huber (2007).

${ }^{7}$ There is also a notion of incremental evidential support in ranking theory. See Spohn (2009: 194ff).
} 
$R(\neg e)=\infty$, although weaker notions of evidence are available, such as the logically strongest proposition the agent believes, or the logically strongest proposition the agent believes with sufficient strength.

Absolute evidential support is internally accessible for the agent, because it is defined exclusively in terms of the agent's rational system of full beliefs. If the agent's system of full beliefs is rational in the sense that it is a ranking function, and if $e$ is the agent's evidence, i.e. is the logically strongest proposition the agent is certain of in the sense of assigning its negation rank $\infty$, then the agent's rational strength of disbelief in any proposition $h$ is her degree of absolute evidential support for $h$ given $e$. In this sense the agent believes in accordance with her evidence. Thus, whether it is rational to believe in accordance with one's evidence depends on whether ranking functions really are rational systems of full belief. This in turn depends on the existence of an appropriate means-end relationship between being a ranking function and having true beliefs. Logical entailment is such an appropriate means-end relationship, and this means-end relationship is exactly what the consistency argument establishes.

The conclusion of the consistency argument is the norm of ranking theory that an agent's system of full beliefs should obey the (synchronic and diachronic rules of the) ranking calculus. An agent's system of full beliefs is said to be diachronically consistent if and only if it is and will always be consistent and deductively closed. Thus, diachronic consistency is the "eternal" version of the conjunction of consistency and deductive closure at a given moment in time. It requires an agent's system of full beliefs to be "synchronically consistent", i.e. consistent and deductively closed, at any given moment in time, and to remain so when new information is received and the system is updated. The requirement of diachronic consistency is non-trivial in the sense that it does not allow an agent to move to an arbitrary new system of full beliefs that is consistent and deductively closed, because it also applies to the agent's conditional beliefs. For instance, upon becoming certain that $p$ an agent cannot simply move from believing $q$ and disbelieving $\neg q$ to disbelieving $q$ and believing $\neg q$ without also considering the conditional beliefs in $q$ given $p$ and $\neg q$ given $p$. The core of the argument is a theorem to the effect that an agent's system of full beliefs obeys 
the (synchronic and diachronic rules of the) ranking calculus if and only if it is diachronically consistent. Thus, obeying the norm of ranking theory is a (provably necessary and sufficient) means to attaining the end of having a diachronically consistent system of full beliefs. In other words, the norm of ranking theory has a means-end justification with respect to the goal of having a diachronically consistent system of full beliefs. Since the diachronic consistency of a system of full beliefs is a necessary condition for the joint truth of all the beliefs in the system at every moment in time, the consistency argument establishes a (provably necessary) relationship between the norm of ranking theory and the goal of "eternal" truth. ${ }^{8}$

For ease of reference let us repeat the second non-categorically normative notion of evidential support that we have identified.

(E2) absolute evidential support in the sense of a ranking function $R$, which is interpreted as the agent's rational system of full belief: $r$ is the agent's degree of absolute evidential support for a proposition $h$ provided by the agent's evidence $e$ if and only if $r$ is the agent's rational strength of disbelief for $\neg h$ given $e, r=R(\neg h \mid e)$, where the agent's evidence $e$ is the logically strongest proposition the agent is certain of in the sense of assigning its negation rank $\infty$. In particular, a proposition $h$ is absolutely supported by the agent's evidence $e$ if and only if $h$ is rationally believed given $e, R(\neg h \mid e)>0$.

This notion of evidential support is hypothetically normative. It is defined to be identical with a ranking function which is interpreted as a rational system of full beliefs. Therefore, in order to justify the norm that one ought to believe in accordance with one's evidence, it is necessary and sufficient to provide a means-end justification for the norm of ranking theory. Since obeying the norm of ranking theory is a provably necessary and sufficient means to attaining the end of having a system of full beliefs that is diachronically consistent, which in turn is a necessary condition for "eternal" truth, i.e. the

\footnotetext{
${ }^{8}$ Strictly speaking diachronic consistency is only necessary for the joint truth of all the beliefs in the system and their logical consequences at every moment in time. We ignore this complication in order to avoid a discussion of how to represent the contents of beliefs and the precise formulation of the goal of having true beliefs.
} 
joint truth of all the beliefs in the system at every moment in time, believing in accordance with one's evidence is a provably necessary means to attaining the end of having true beliefs.

Whichever, if any, of these goals one de facto has, the point to note is that the normative notions of a rational system of full beliefs and of a rational system of degrees of belief have means-end justifications with respect to the default cognitive goals of believing the truth and believing accurately, and so can be understood in instrumentalist terms. More precisely, by expressing the terminal parameter of being true or at least diachronically consistent the normative notion of a rational system of full beliefs becomes the descriptive notion of a system of full beliefs that is a provably necessary (and sufficient) means to attaining the end of being true (or diachronically consistent). Similarly, by expressing the terminal parameter of being accurate or at least not accuracy dominated the normative notion of a rational system of degrees of belief becomes the descriptive notion of a system of degrees of belief that is a provably necessary (and sufficient) means to attaining the end of being accurate (or not accuracy dominated).

Consequently the normative notions of absolute evidential support that are defined in terms of rational systems of full beliefs and rational systems of degrees of belief are hypothetically normative, and so epistemic rationality is a form of instrumental rationality. The reason for this is the following. In Bayesianism absolute evidential support is defined exclusively in terms of an agent's rational degree of belief function, and in ranking theory absolute evidential support is defined exclusively in terms of an agent's rational system of full beliefs. This is why absolute evidential support is internally accessible. On these accounts the link between an agent's degree of belief function or system of full beliefs and evidential support is defined to be identity. This is why an agent believes in accordance with her evidence if and only if her degree of belief function or system of full beliefs is a probability measure or ranking function, respectively. Therefore, once the norm of probabilism and the norm of ranking theory are given a means-end justification, the norm that an agent ought to believe in accordance with her evidence is automatically given the same means-end justification. We conclude that absolute evidential support saves the instrumentalist conception of epistemic rationality by being 
a hypothetically normative notion.

\section{Acknowledgments}

We are very grateful to James M. Joyce and an anonymous referee as well as Michael Baumgartner, Luke Glynn, Brian Leahy, Christoph Schmidt-Petri, and Wolfgang Spohn for many helpful comments and suggestions.

Our research was supported by the German Research Foundation through its Emmy Noether Program and the Zukunftskolleg at the University of Konstanz.

\section{References}

[1] Carnap, Rudolf (1962), Logical Foundations of Probability. Chicago: The University of Chicago Press.

[2] Field, Hartry (1978), A Note on Jeffrey Conditionalization. Philosophy of Science 45, 361-367.

[3] Huber, Franz (2007), The Consistency Argument for Ranking Functions. Studia Logica 86, 299-329.

[4] Jeffrey, Richard C. (1983), The Logic of Decision. 2nd ed. Chicago: University of Chicago Press.

[5] Joyce, James M. (1998), A Nonpragmatic Vindication of Probabilism. Philosophy of Science 65, 575-603.

[6] Joyce, James M. (2009), Accuracy and Coherence: Prospects for an Alethic Epistemology of Partial Belief. In F. Huber and C. SchmidtPetri (eds.), Degrees of Belief. Dordrecht: Springer, 263-300.

[7] Kelly, Thomas (2003), Epistemic Rationality as Instrumental Rationality: A Critique. Philosophy and Phenomenological Research 66, 612640 . 
[8] Kelly, Thomas (2007), Evidence and Normativity: Reply to Leite. Philosophy and Phenomenological Research 75, 465-474.

[9] Leite, Adam (2007), Epistemic Instrumentalism and Reasons for Belief: A Reply to Thomas Kelly 'Epistemic Rationality as Instrumental Rationality: A Critique'. Philosophy and Phenomenological Research 75, 456-464.

[10] Percival, Philip (2002), Epistemic Consequentialism. Proceedings of the Aristotelian Society: supplementary volume 76, 121-151.

[11] Quine, Willard van Orman (1986), Reply to Morton White. In L. Hahn and P. Schilpp (eds.), The Philosophy of W. V. Quine. La Salle: Open Court.

[12] Ramsey, Frank P. (1926), Truth and Probability. In R. B. Braithwaite (ed.), Foundations of Mathematics and other Essays. London: Routledge \& P. Kegan, 1931, 156-198.

[13] Spohn, Wolfgang (1988), Ordinal Conditional Functions: A Dynamic Theory of Epistemic States. In W.L. Harper \& B. Skyrms (eds.), Causation in Decision, Belief Change, and Statistics. Vol. II. Dordrecht: Kluwer, 105-134.

[14] Spohn, Wolfgang (2009), A Survey of Ranking Theory. In F. Huber and C. Schmidt-Petri (eds.), Degrees of Belief. Dordrecht: Springer, 185-228.

[15] Stalnaker, Robert (2002), Epistemic Consequentialism. Proceedings of the Aristotelian Society: supplementary volume 76, 153-168.

[16] van Fraassen, Bas C. (1983), Calibration: A Frequency Justification for Personal Probability. In R. Cohen \& L. Laudan (eds.), Physics, Philosophy, and Psychoanalysis. Dordrecht: D. Reidel, 295-319. 